\title{
A simple device for producing synchronous and asynchronous binocular flicker
}

\author{
ARNULF REMOLE and DOUGLAS GREGORY \\ School of Optometry, University of Waterloo, Waterloo, Ontario, Canada N2L $3 G 1$
}

\begin{abstract}
Many studies on binocular integration have compared in-phase and out-of-phase flicker stimulation. A new method of producing such stimulation is described. It differs from previous designs in that a combination of polarizers is used rather than mechanical light choppers or electronic flashers. The arrangement produces sinusoidal luminance pulses that can be presented with any degree of asynchrony between the two eyes.
\end{abstract}

The study of binocular flicker stimulation originated with Sherrington (1947), who compared monocular and binocular flicker fusion thresholds as well as in-phase and out-of-phase binocular fusion thresholds. With Sherrington's original apparatus, the flicker stimulation was produced by a perforated vertical drum rotating about an incandescent lamp. The perforations were arranged in tiers, one above the other, that could be adjusted independently so as to pass through the field of view in step or out of step with the two eyes. A shutter and prism arrangement permitted fusion of the flickering stimulus fields. Thus, binocular synchronous flicker, as well as monocular flicker, could be presented. Two stimulus combinations could be presented simultaneously, one appearing above the other.

Baker (1952) used two revolving sectored disks that alternately exposed and occluded pairs of luminous targets. As with Sherrington's apparatus, two stimulus situations could be viewed simultaneously, one above the other. Perrin (1954) presented only one stimulus situation at a time; this could be monocular, binocular synchronous, or binocular with varying degrees of asynchrony. A single chopper in combination with two light sources produced synchronous flashing when its center was moved along the right bisector to this line, the degree of asynchrony depending on the amount of this displacement. To insure instantaneous occlusion, the sources were focused in the ocular entrance pupils by appropriate lens systems. Ireland (1950) used two lamps, one for each eye, energized by an electronic pulse generator. By changing the phase relationship of the impulses applied to the two lamps, they could be made to flash in phase or out of phase with one another. Rectangular pulses were produced as with the other designs. Again, only one stimulus combination was presented at a time.

The apparatus presented in this report differs from previous designs in that it utilizes a combination of polarizing filters. A single target is viewed binocularly through the rotating polarizer. When the plane-polarized light passes through an analyzer mounted before each eye, a series of sinusoidal luminance fluctuations is produced. If the axes of the analyzers are parallel, the pulses will be in step; if an angle is introduced between the axes, the pulses will be out of step.

The apparatus is shown schematically in Figure 1; apparatus components are listed in Table 1 . The single field, viewed binocularly through the polarizing filter combination, consists of an aperture $3 \mathrm{~cm}$ in diam exposing a transilluminated diffusing Plexiglas sheet.

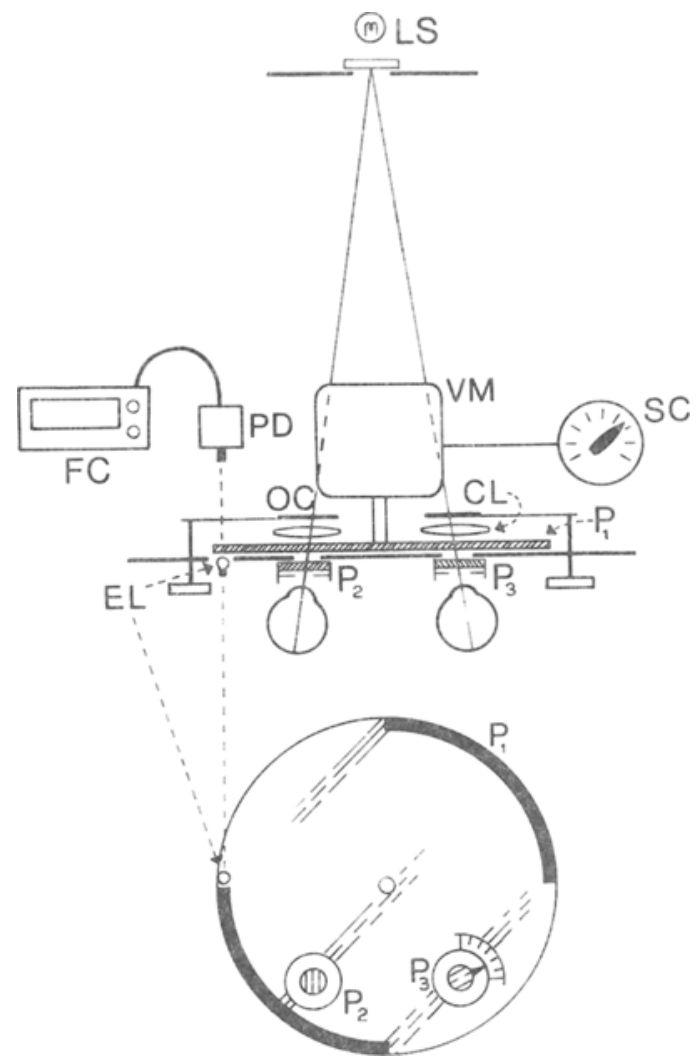

Figure 1. Schematic illustration of apparatus. LS, light source and diffusing stimulus field; $P_{1}$, rotating polarizer; $P_{2}$, left eye analyzer; $P_{3}$, right eye adjustable analyzer with scale showing axis orientation; $V M$, variable-speed motor with SC, speed control; OC, occluders; CL, correcting lenses or prisms for focusing target; PD, photodiode with sensor; EL, excitor lamp; FC, frequency counter. 
Table 1

Specifications of Apparatus Components

\begin{tabular}{ll}
\hline $\begin{array}{l}\text { Polarizing } \\
\text { filters }\end{array}$ & $\begin{array}{l}\text { No. } 71939 \text { polarizing film, supplied by Edmund } \\
\text { Scientific Company }\end{array}$ \\
$\begin{array}{l}\text { Electric } \\
\text { motor }\end{array}$ & $\begin{array}{l}115-\mathrm{V} \text { ac } 1 / 15-\mathrm{HP} \text { motor, Model 6705, supplied } \\
\text { by Electrohome Ltd. }\end{array}$ \\
$\begin{array}{l}\text { Motor speed } \\
\text { control }\end{array}$ & $\begin{array}{l}\text { Variable autotransformer, supplied by Staco, } \\
\text { Inc. }\end{array}$ \\
$\begin{array}{l}\text { Frequency } \\
\text { counter }\end{array}$ & BK precision frequency counter, Model 1801, \\
shotodiode & Nopplied by Dynascan Corporation \\
& $\begin{array}{l}\text { Communications } \\
\text { Luminance }\end{array}$ \\
Control & Variable autotransformer, supplied by Staco, \\
\hline
\end{tabular}

Illumination is provided by a $100-\mathrm{W}$ incandescent bulb, whose luminous output is controlled by a transformer. This can readily be replaced by a dc source if the small ac fluctuations interfere with the pulses produced by the polarizers. In our application, this was unnecessary. If difficulties are experienced in viewing the field binocularly, appropriate lenses or prisms can be placed in the lines of sight.

The rotating polarizer is driven by a $1 / 15-\mathrm{HP}$ variablespeed motor connected to a transformer. The motor speed can be controlled by a dial operated by the subject. The polarizer disk, $16 \mathrm{~cm}$ in diam, simultaneously interrupts both lines of sight when directed toward the stimulus field. The disk consists of polarizing film laminated between two sheets of 1/16-in.-gauge Plexiglas. Two cycles of transmission and occlusion occur for each disk revolution. The disk is mounted quite close to the face plane. However, unlike a design based on a mechanical chopper, the position of the polarizer disk in relation to the light source or the ocular entrance pupils is immaterial and does not affect the shape of the pulses. Thus, the polarizer can be placed immediately in front of the stimulus field. The alternating clear and opaque concentric bands at the edge of the disk intercept light from a small excitor lamp directed at the sensor of a photodiode. The photodiode is connected to a Dynascan 1801 frequency counter, which registers, in cycles per second, the changes in the plane of polarization. In Figure 1, the axis of the polarizer is shown by sets of partially broken lines.

The analyzers are mounted in holders with 3-mmdiam artificial pupils. The holders can be translated along the horizontal meridian of the face plane so as to match the subject's interpupillary distance during fixation of the target. The right eye analyzer can be rotated through $90 \mathrm{deg}$ so as to vary the angle between the axes of the analyzers, thus producing any desired amount of asynchrony between the left and right eye stimuli. The rotation scale is calibrated in 15-deg increments. In Figure 1, the directions of the axes of the analyzers are shown by the parallel lines across the apertures. The placement of the analyzer apertures in relation to the center of the polarizing disk is immaterial, for it does not affect the angular relationship between polarizer and analyzer axes.

Figure 2 shows an oscilloscope tracing of the nearly sinusoidal luminous pulses produced by the apparatus. Some small imperfections are evident, for example, the slight differences in height of pulses produced by opposite sides of the polarizer. These are probably due to slight differences in performance between various portions of the polarizer. The differences between monocular and binocular flicker fusion thresholds, and between synchronous and asynchronous fusion thresholds, appear to be of the same order and magnitude with sinusoidal pulses as with rectangular pulses.

The principle of utilizing polarizers to produce temporal intensity variations is not new. A rotating polarizer in combination with analyzers arranged in squares, as in a checkerboard pattern and with their axes orthogonal, has been used for making alternating patterns that serve as stimuli during visually evoked electrophysiological recordings (Behrman, Nissim, \& Arden, 1972; Millodot \& Riggs, 1970; Riggs, 1974). However, to our knowledge, using polarizers to present alternating stimuli to the two eyes is a new application. The apparatus described here is simple, smooth, and relatively noiseless. No pulleys or gears are needed, as in Sherrington's original system, and only one stimulus field is necessary. Also, the instrument does not present the potential problem of unequal illumination of the two eyes that exists when two optical systems or two light sources are used.

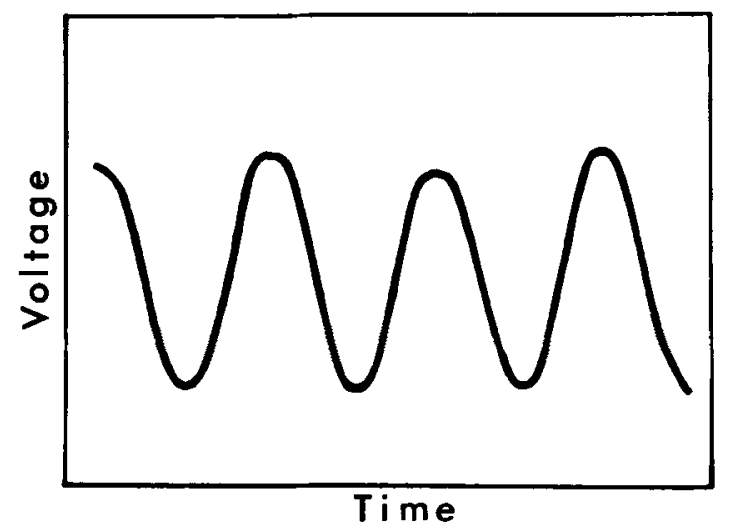

Figure 2. Oscilloscope tracing of light transmitted by one of the analyzers during the rotation of the polarizer disk.

\section{REFERENCES}

BAKER, C. H. The dependence of binocular fusion on timing of peripheral stimuli and on central process. Canadian Journal of Psychology, 1952, 6, 1-10.

Behrman, J., Nissim, S., \& Aroen, G. B. A clinical method for obtaining pattern visual evoked responses. Advances in Experimental Medicine and Biology, 1972, 24. 199-206. 
IRELAND, F. H. A comparison of critical tlicker frequencies under conditions of monocular and binocular stimulation. Journal of Experimental Psychology, 1950, 40, 282-285.

Millodot, M., \& Riggs, L. A. Refraction determined electrophysiologically. Archives of Ophthalmology, 1970, 84, 272-278.

Perrin, F. H. A study in binocular flicker. Journal of the Optical Society of America, 1954, 44, 60-69.

Riggs, L. A. Responses of the visual system to fluctuating patterns. American Journal of Optometry and Physiological Optics, 1974, 51, 725-735.

Sherrington, C. S. Lecture 10: Sensual fusion. The integrative action of the nervous system. New Haven: Yale University Press, 1947.

(Received for publication March 15, 1977; revision accepted April 26. 1977.) 